\title{
Diagnostic value of candidate noncoding RNAs in leukocytes of patients with gestational diabetes mellitus
}

\author{
JIALEI HU ${ }^{1 *}$, HANYOU MU ${ }^{1 *}$, LINSHAN GAO ${ }^{1}$, YING PAN ${ }^{1}$, CHUANFEI WU $^{2}$, \\ DONG ZHANG $^{1}$, QIAOJUN CHEN ${ }^{1}$ and HONGHUI DING ${ }^{1}$ \\ ${ }^{1}$ Clinical Laboratory, Yiwu Maternal and Child Health Hospital; ${ }^{2}$ Department of Pediatrics, \\ Yiwu Maternal and Child Health Hospital, Jinhua, Zhejiang 321000, P.R. China
}

Received February 3, 2020; Accepted July 29, 2020

DOI: $10.3892 / \mathrm{etm} .2020 .9576$

\begin{abstract}
Noncoding RNAs (ncRNAs) are involved in the pathological processes of various diseases. The aim of the present study was to verify the expression levels and the diagnostic value of two candidate ncRNAs in the blood leukocytes of patients with gestational diabetes mellitus (GDM) compared to healthy controls. The long ncRNA paired box 8 antisense 1 (Pax8-AS1) and the microRNA miR-4646 were selected, which were identified to be associated with GDM by bioinformatics analysis of a dataset from the Gene Expression Omnibus GEO database. By using reverse transcription-quantitative PCR, the expression levels of Pax8-AS1 and miR-4646 were analysed in leukocytes of patients with GDM $(n=35)$ and normal pregnant females $(n=35)$. The results indicated a significant decrease in the expression levels of both Pax8-AS1 and miR-4646 in patients with GDM as compared with those in the healthy controls. In the second trimester, a strong negative correlation between Pax8-AS1/miR-4646 and 2-h glucose levels was detected in patients with GDM. Receiver operating characteristic curve analysis indicated that the levels of Pax8-AS1 and miR-4646 in the second trimester of pregnancy had a significant diagnostic value with high selectivity and specificity for GDM (area under the curve values, 0.902 and 0.891 , respectively; $\mathrm{P}<0.001$ ). Overall, the present study suggested that Pax8-AS1 and miR-4646 may serve as promising diagnostic biomarkers for GDM.
\end{abstract}

Correspondence to: Professor Qiaojun Chen or Dr Honghui Ding, Clinical Laboratory, Yiwu Maternal and Child Health Hospital, C100 Xinke Road, Jinhua, Zhejiang 321000, P.R. China

E-mail: qiaojunywfb@163.com

E-mail: honghui0189@163.com

*Contributed equally

Key words: gestational diabetes mellitus, long noncoding RNAs, microRNAs

\section{Introduction}

Gestational diabetes mellitus (GDM) is the most common medical complication of pregnancy and is known as different degrees of glucose intolerance that are initially identified during pregnancy (1). Owing to the increase in the rate of obesity and the increase in maternal age, the morbidity of gestational diabetes is currently increasing (2). However, the pathogenesis of GDM remains to be fully elucidated and early-mid pregnancy diagnostic markers may facilitate progress in this area. Controversy persists regarding GDM screening, but current consensus recommendations support screening for GDM during mid-pregnancy (24-28 weeks of gestation) (3). In addition to its detrimental effects on maternal health, GDM has also been demonstrated to impede foetal health and amplify the offspring's tendency to develop metabolic diseases, such as obesity and type 2 diabetes mellitus (T2D) later in life (4-6). Therefore, the identification of novel signatures or biomarkers that enhance the determination of clinical behaviours is essential for the treatment of GDM.

Several studies have reported that there are multiple genes associated with diabetes. Over the past decades, several types of non-coding RNA (ncRNA) species, including microRNAs (miRNAs/miRs), long ncRNAs (lncRNAs) and circular RNAs, have emerged as pivotal regulators of multiple cellular functions, including cell proliferation, apoptosis, cellular differentiation, tumorigenesis and metastasis (7). LncRNAs are defined as RNAs with a length of $>200$ nucleotides and no protein-coding ability $(8,9)$. Numerous IncRNAs have been reported to be involved in GDM progression. Among them, the lncRNA metastasis associated in lung adenocarcinoma transcript 1 (MALAT1) was increased in endothelial cells after high levels of glucose stimulation. Mechanistic investigations suggested that MALAT1 promotes insulin resistance by increasing the stability of the nuclear transcription factor sterol regulatory element-binding protein $1 \mathrm{C}(10)$. However, to the best of our knowledge, only a small number of studies have been performed on the utility of circulating lncRNAs for the dynamic monitoring of patients for GDM (11).

miRNAs are known to post-transcriptionally regulate the expression of their target genes by binding to the $3^{\prime}$ untranslated region of the respective mRNA (12). The identification of stable circulating miRNAs in plasma and serum has provided 
promise for minimally invasive biomarkers for disease prediction, diagnosis and prognosis (13). According to a previous review, an increasing number of studies have identified that certain miRNAs are associated with insulin secretion, inflammation and insulin resistance (14). Differences have also been detected in circulating levels of miRNAs when comparing T2D cases to controls (15).

In the present study, bioinformatics analysis was used to explore significantly differentially expressed genes during GDM progression and the lncRNA paired box 8 antisense 1 (PAX8-AS1) and miR-4646 were identified as the most downregulated ncRNAs in GDM. Therefore, the study further endeavoured to investigate PAX8-AS1 and miR-4646 in the blood leukocytes of patients with GDM compared to those of healthy pregnant females as biomarkers for the diagnosis and monitoring of GDM.

\section{Materials and methods}

Retrieval of RNA sequencing data and analysis. RNA sequencing datasets from the gene expression omnibus (GEO) database were used to analyse the expression profiles of lncRNAs and miRNAs in GDM (www.ncbi.nlm.nih.gov/geo). The dataset no. GSE92772 was used, containing the expression data of 8 subjects with normal glucose tolerance (NGT) and 8 patients with GDM who were matched in terms of body mass index (BMI) and age (16). The RNA sequencing of the leukocytes of these samples was performed on an Illumina HiSeq 2500 platform (Illumina, Inc.).

Participants and maternal blood collection. To validate the expression levels of IncRNAs and miRNAs in patients with GDM and normal controls, a group of 35 pregnant females with NGT (age, $32 \pm 3$ years; BMI, $27.2 \pm 3.8 \mathrm{~kg} / \mathrm{m}^{2}$ ) and 35 patients with GDM (age, $34 \pm 4$ years; BMI, $28.5 \pm 4.1 \mathrm{~kg} / \mathrm{m}^{2}$ ) were selected from a pregnancy cohort. Maternal blood samples were from the second trimester (24-28 weeks). Patients with other pregnancy-associated diseases, including chronic hypertension, multiple pregnancies or any other diseases, including liver or kidney disease, cancer and gynaecological diseases, were excluded from the present study. Serum and blood samples of the pregnant females in the second trimester (24-28 weeks of gestation) were collected at the Yiwu Women's and Children's Hospital (Jinhua, China) between June and November 2019. Patients who had other pregnancy-associated diseases, such as gynaecological diseases, or carcinomas, chronic hypertension or liver diseases, were excluded from the present study.

Oral glucose tolerance test and clinical chemical analyses. The participants underwent a 5-point 75-g oral glucose tolerance test after overnight fasting. Venous blood samples were obtained at 0,60 and $120 \mathrm{~min}$ for the determination of plasma glucose and insulin levels. Blood glucose was determined using a bedside glucose analyser (Roche Diagnostics). NGT was defined as fasting glucose $\leq 5.11 \mathrm{mmol} / 1$, 1-h glucose $\leq 10.00 \mathrm{mmol} / \mathrm{l}$ and 2 -h glucose $\leq 8.50 \mathrm{mmol} / \mathrm{l}$. GDM patients were diagnosed according to the recommendations of the International Association of the Diabetes and Pregnancy Study Groups for the diagnosis and classification of hyperglycaemia during pregnancy, published in 2010 (3).
Total RNA extraction and reverse transcription-quantitative $(R T-q) P C R$. Firstly, to separate leukocytes from the blood samples, $200 \mu \mathrm{l}$ fresh blood was taken, where $600 \mu \mathrm{l}$ red blood cell lysate buffer (cat. no. R1010; Beijing Solarbio Science \& Technology Co., Ltd.) was added and mixed at room temperature for $10 \mathrm{~min}$, which were then and centrifuged for $7300 \mathrm{x} \mathrm{g}$ for $1 \mathrm{~min}$ at room temperature. Leukocytes were obtained after supernatant was discarded. Total RNA from maternal blood leukocytes was then extracted using TRIzol ${ }^{\circledR}$ reagent according to manufacturer's protocol (Invitrogen; Thermo Fisher Scientific, Inc.). Complementary (c)DNA was synthesized with PrimeScript ${ }^{\mathrm{TM}}$ IV 1st strand cDNA Synthesis Mix (Takara Biotechnology, Co., Ltd.) using a LightCycler 480 II (Roche Diagnostics). The reverse transcription was performed under the following conditions: $30^{\circ} \mathrm{C}$ for $10 \mathrm{~min}$, followed by $42^{\circ} \mathrm{C}$ for $15 \mathrm{~min}$. Real-time PCR analyses were quantified using TB Green ${ }^{\circledR}$ Fast qPCR Mix (Takara Biotechnology, Co., Ltd.) on a LightCycler 480 II (Roche Diagnostics). The PCR reaction system is as follows: $4 \mu \mathrm{l}$ qPCR mix, $1 \mu \mathrm{l}$ Taq enzyme, $4 \mu \mathrm{l}$ primers, $2 \mu \mathrm{g}$ cDNA and $10 \mu \mathrm{l}$ water. The following candidate ncRNAs were selected: miR-4646, Pax8-AS1, miR-5196, interleukin 21 receptor-AS1 (IL21R-AS1), signal recognition particle 14-AS1 (SRP14-AS1), MORC family CW-type zinc finger 2-AS1 (MORC2-AS1), miR-8061, LIM homeobox 4-AS1 (LHX4-AS1), Sjogren syndrome/scleroderma autoantigen 1-AS1 (SSSCA1-AS1) and miR-3679. The sequences of the primers used are listed inTable I. The amplification was performed under the following conditions: $95^{\circ} \mathrm{C}$ for $10 \mathrm{~min}$ for initial denaturation; followed by 35 cycles of $94^{\circ} \mathrm{C}$ for $15 \mathrm{sec}$, annealing at $56^{\circ} \mathrm{C}$ for $25 \mathrm{sec}$ and $72^{\circ} \mathrm{C}$ for $30 \mathrm{sec}$. The expression levels of a gene were determined by the following formula: $\Delta \mathrm{Cq}=\mathrm{Cq}$ (target gene)- $\Delta \mathrm{Cq}$ (internal reference gene) (17). The levels of the ncRNAs were normalized to the levels of U6 (18).

Statistical analysis. SPSS 18.0 software (SPSS, Inc.) was used to statistically analyse the data. Receiver operating characteristic (ROC) curve analysis was applied to determine the best cut-off values of Pax8-As1 and miR-4646 for diagnosing patients with GDM. The Youden index is used to determine the best cut-off value (19). Correlation between two variables was analyzed using the Pearson's correlation test. Independent-samples unpaired t-tests were used when a normal distribution and homogeneity of variance were both satisfied. $\mathrm{P}<0.05$ was considered to indicate statistical significance.

\section{Results}

Different expression patterns in blood leukocytes of patients with GDM. To identify essential ncRNAs in GDM, raw microarray data were downloaded from GEO (dataset no. GSE92772). To obtain differentially expressed genes, the signal data were normalized and $\mathrm{z}$-score-transformed (unpaired t-test according to the experimental design was used to validate statistical significance; Fig. 1A). It was determined that numerous lncRNAs and miRNAs were decreased in patients with GDM compared to normal controls. Among them, miR-4646 and Pax8-AS1 were the most downregulated and conserved ncRNAs in the GSE92772 dataset (Fig. 1B).

To explore the difference between the GDM and normal gravidas, a comprehensive metabolic panel in the maternal serum 
Table I. Primer sequences used for PCR.

\begin{tabular}{lll}
\hline Gene & \multicolumn{1}{c}{ Forward primer $\left(5^{\prime} \rightarrow 3^{\prime}\right)$} & Reverse primer $\left(5^{\prime} \rightarrow 3^{\prime}\right)$ \\
\hline miR-4646 & ACTGGGAAGAGGAGCT & TGTCGTGGAGTCGGC \\
Pax8-AS1 & CCCCAAAGCCTAACTCCCTG & CACTTGGGTTTTGCTGCCTC \\
miR-5196 & TCTGAGGAGACCTGGGCTGT & TGTCGTGGAGTCGGC \\
IL21R-AS1 & TGCTGGTTCTTGTAGCTCCG & CTATGGGGCCACCAGTTGTC \\
SRP14-AS1 & CTAACTCTGCCACACACGGT & GCTCAGACCTGCAACCTCTT \\
MORC2-AS1 & GTTCATAACCGTTGGCTGGT & GGGTCCACTTTTGTCCCCAA \\
miR-8061 & GAGAGGATGCCTTAGATTA & TGTCGTGGAGTCGGC \\
LHX4-AS1 & GCATCTCACCTGTACGACCC & TGCTGCAGATAGGCCGAAG \\
SSSCA1-AS1 & CCATCGCCACCCCAGTAATC & CATGCAGAAGGGGAGTGGTC \\
U6 6 C 19 & CGTGGTGAGGATATGGCAGG & TGTCGTGGAGTCGGC \\
\end{tabular}

miR, microRNA; Pax8-AS1, paired box 8 antisense 1; IL21R, interleukin 21 receptor; SRP14, signal recognition particle 14; MORC2, MORC family CW-type zinc finger 2; LHX4, LIM homeobox 4; SSSCA1, Sjogren syndrome/scleroderma autoantigen 1.

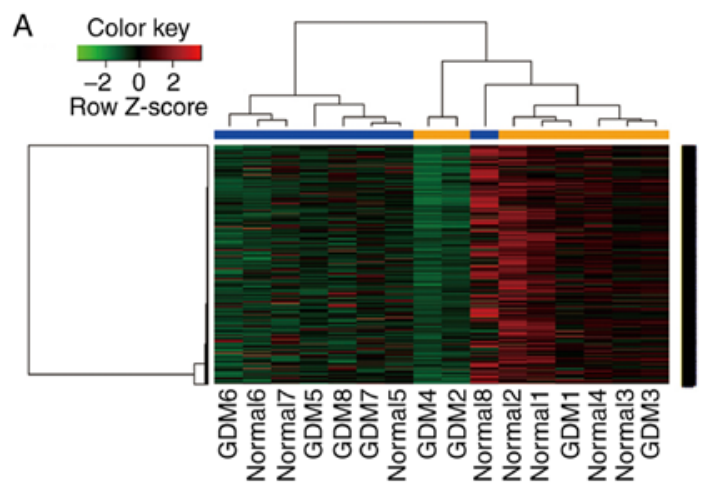

B

\begin{tabular}{|c|}
\hline Top 10 down-regulated non-coding RNAs \\
\hline miR-4646 Pax8-AS1 \\
miR-5196 IL21R-AS1 SRP14-AS1 MORC2-AS1 \\
miR-8061 LHX4-AS1 SSSCA1-AS1 miR-3679 \\
\hline
\end{tabular}

Figure 1. Identification of differentially expressed genes in GDM. (A) Differentially expressed genes were detected by analysing the gene expression omnibus dataset GSE92772. The heatmap of all differentially expressed genes is presented. (B) Top 10 downregulated ncRNAs in GDM. GDM1-6, cases; normal1-8, controls. GDM, gestational diabetes mellitus; miR, microRNA; Pax8-AS1, paired box 8 antisense 1; IL21R, interleukin 21 receptor; SRP14, signal recognition particle 14; MORC2, MORC family CW-type zinc finger 2; LHX4, LIM homeobox 4; SSSCA1, Sjogren syndrome/scleroderma autoantigen 1.

samples was tested. The results of the oral glucose tolerance test were significantly higher in the GDM group than in the control group $(\mathrm{P}<0.05)$. However, there was no significant difference in other laboratory indicators, including uric acid (UA), total bile acid (TBA), globulin (GLOB), alanine aminotransferase or aspartate aminotransferase, between the cases and controls (Table II).

ncRNA expression patterns in blood leukocytes associated with GDM. To explore the expression of 10 candidate GDM-associated ncRNAs in blood leukocytes, the total
RNA from whole blood cells was extracted from 35 subjects with NGT and 35 patients with GDM. As indicated in Fig. 2, the levels of miR-4646, Pax8-AS1, miR-5196, IL21R-AS1, SRP14-AS1, LHX4-AS1, SSSCA1-AS1 and miR-3679 in patients with GDM were significantly lower than those in the controls. However, there were no significant differences in the levels of MORC2-AS1 and miR-8061 between the two groups. Furthermore, miR-4646 and Pax8-AS1 were the most downregulated ncRNAs among them. Subsequently, the correlations between the expression levels of the eight candidate genes (miR-4646, Pax8-AS1, miR-5196, IL21R-AS1, SRP14-AS1, LHX4-AS1, SSSCA1-AS1 and miR-3679) and 2-h glucose in the leukocytes of patients with GDM were detected. As presented in Fig. 3, a significant negative correlation was observed between miR-4646, Pax8-AS1, miR-5196 and SRP14-AS1 levels and 2-h glucose in the GDM group $(r=0.83, r=0.73, r=0.54$ and $r=0.64$, respectively; $\mathrm{P}<0.001)$.

ROC analysis. To determine the diagnostic accuracy of miR-4646, Pax8-AS1, miR-5196 and SRP14-AS1 in maternal blood leukocytes as biomarkers for GDM, ROC curves were drawn (Fig. 4). The area under the ROC curve (AUC) was used to assess the diagnostic value of ncRNAs for GDM. In the ROC analysis, miR-4646 and Pax8-AS1 achieved an obvious separation between the GDM and control groups. The AUC of miR-4646 during the second trimester was 0.891 (95\% CI: $0.811-0.972, \mathrm{P}<0.001)$; its highest sensitivity and specificity were 88.6 and $82.9 \%$, respectively (cut-off, 1.95). The AUC of Pax8-AS1 for GDM was 0.902 (95\% CI: 0.824-0.979, $\mathrm{P}<0.001)$, with a sensitivity and specificity of 85.7 and $88.6 \%$, respectively (cut-off, 2.165).

\section{Discussion}

GDM is common and frequently has serious consequences for mothers and their children. The roles of several ncRNAs in the occurrence of GDM have recently attracted our attention, namely miRNAs and lncRNAs. To determine the role of ncRNAs in GDM, circulating miRNAs and lncRNAs from 
Table II. Variables of the comprehensive metabolic panel associated with GDM.

\begin{tabular}{|c|c|c|c|}
\hline Variable & Normal range & Normal glucose tolerance & GDM \\
\hline $\operatorname{ALT}(\mathrm{U} / \mathrm{l})$ & $7-45$ & $16.83 \pm 3.78$ & $14.75 \pm 2.34$ \\
\hline $\operatorname{AST}(\mathrm{U} / 1)$ & $13-40$ & $15.83 \pm 4.26$ & $17.16 \pm 5.09$ \\
\hline $\mathrm{DBIL}(\mu \mathrm{mol} / \mathrm{l})$ & $0.0-8.0$ & $1.95 \pm 0.59$ & $1.75 \pm 0.38$ \\
\hline TBIL $(\mu \mathrm{mol} / 1)$ & $0.0-21.0$ & $4.65 \pm 1.18$ & $4.67 \pm 0.94$ \\
\hline CREA $(\mu \mathrm{mol} / 1)$ & $41-73$ & $46.33 \pm 5.74$ & $48.45 \pm 6.15$ \\
\hline $\mathrm{UA}(\mu \mathrm{mol} / \mathrm{l})$ & $142-339$ & $260.08 \pm 67.11$ & $247.55 \pm 69.14$ \\
\hline $\mathrm{TBA}(\mu \mathrm{mol} / \mathrm{l})$ & $0.0-12.0$ & $2.29 \pm 1.07$ & $2.50 \pm 1.32$ \\
\hline GLOB (g/l) & $20.0-40.0$ & $25.91 \pm 1.79$ & $25.9 \pm 2.47$ \\
\hline ALB (g/l) & $40.0-55.0$ & $38.64 \pm 1.96$ & $37.58 \pm 2.64$ \\
\hline $\operatorname{ALP}(\mathrm{U} / \mathrm{l})$ & $35-100$ & $88.75 \pm 35.59$ & $108.25 \pm 46.41$ \\
\hline LDH (U/l) & $120-250$ & $175.1 \pm 33.99$ & $174.41 \pm 20.147$ \\
\hline $\operatorname{ADA}(\mathrm{U} / \mathrm{l})$ & $0.0-15.0$ & $8 \pm 1.89$ & $7.36 \pm 1.51$ \\
\hline 2-h glucose (mmol/l) & $<8.5$ & $5.40 \pm 0.75$ & $9.69 \pm 0.96^{\mathrm{a}}$ \\
\hline 1-h glucose (mmol/l) & $<10.0$ & $6.47 \pm 1.02$ & $10.23 \pm 1.69^{\mathrm{a}}$ \\
\hline Fasting glucose (mmol/l) & $<5.1$ & $4.73 \pm 0.82$ & $4.81 \pm 0.82$ \\
\hline
\end{tabular}

${ }^{\mathrm{a}} \mathrm{P}<0.05$; values are expressed as the mean \pm standard deviation. GDM, gestational diabetes mellitus; NGT, normal glucose tolerance; ALT, alanine aminotransferase; AST, aspartate aminotransferase; ALP, alkaline phosphatase; DBIL, Direct Bilirubin; TBIL, total bilirubin; CREA, creatinine; UA, uric acid; GLOB, globulin; ALB, albumin; ALP, alkaline phosphatase; LDH, lactate dehydrogenase; ADA, Adenosine deaminase; LDH, lactate dehydrogenase; TBA, total bile acid.
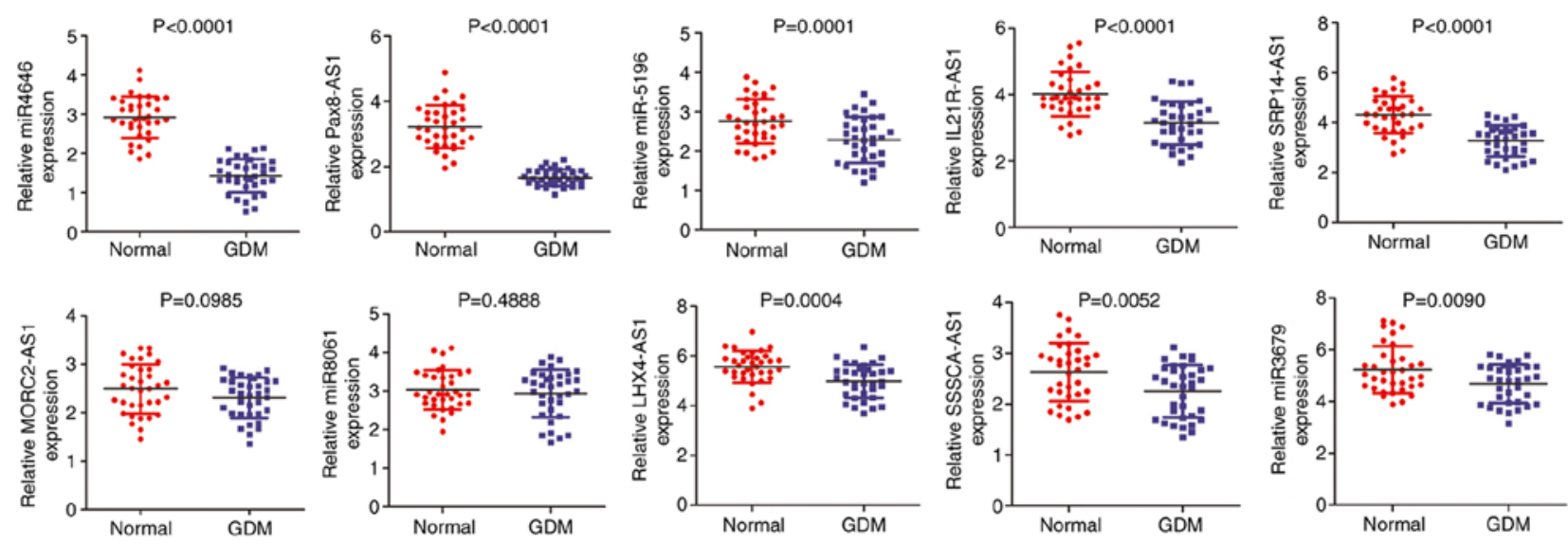

Figure 2. Expression levels of 10 candidate non-coding RNAs in healthy pregnant females (control; $n=35$ ) and patients with gestational diabetes (GDM; $\mathrm{n}=35$ ) during the second trimester were detected by reverse transcription-quantitative PCR. GDM, gestational diabetes mellitus; miR, microRNA; Pax8-AS1, paired box 8 antisense 1; IL21R, interleukin 21 receptor; SRP14, signal recognition particle 14; MORC2, MORC family CW-type zinc finger 2; LHX4, LIM homeobox 4; SSSCA1, Sjogren syndrome/scleroderma autoantigen 1.

maternal blood leukocytes were examined. The present study demonstrated that the levels of Pax8-AS1 and miR-4646 in the blood leukocytes of patients with GDM were significantly downregulated compared with those in pregnant females without GDM. Pax8-AS1 and miR-4646 had the highest diagnostic value for GDM among the differentially expressed ncRNAs identified.

The aberrant expression of lncRNAs is closely associated with the development of multiple complex diseases, including cancer, cardiovascular diseases, nervous system diseases and diabetes (20-22). However, the clinical significance and biological mechanisms of IncRNAs in the progression of GDM have remained largely elusive. In the present study, six lncRNAs were selected as candidate biomarkers and their diagnostic value for GDM was assessed. It was indicated that Pax8-AS1 had the highest diagnostic value for GDM. The lncRNA Pax8-AS1 has been previously reported to participate in the development and progression of diseases, including childhood acute lymphoblastic leukaemia, cervical cancer and papillary thyroid carcinoma (23-25). However, the association between Pax8-AS1 and GDM has remained elusive. The present results indicated that Pax8-AS1 was expressed at low levels in blood leukocytes in the second trimester and was negatively correlated with postprandial blood glucose. ROC curve analysis of ncRNAs from maternal blood leukocytes suggested that the diagnostic value of Pax8-AS1 for GDM was the highest among all lncRNAs tested, 

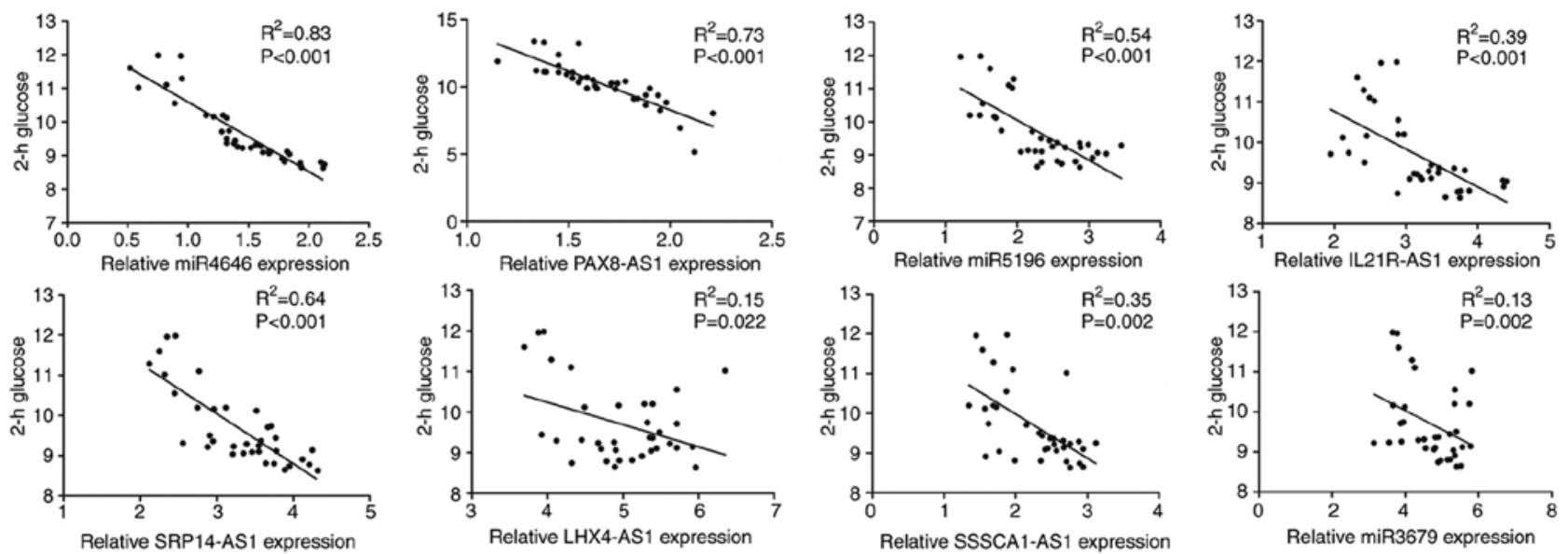

Figure 3. Correlation analysis of ncRNA expression levels with 2-h glucose in the gestational diabetes mellitus group. miR, microRNA; Pax8-AS1, paired box 8 antisense 1; IL21R, interleukin 21 receptor; SRP14, signal recognition particle 14; MORC2, MORC family CW-type zinc finger 2; LHX4, LIM homeobox 4; SSSCA1, Sjogren syndrome/scleroderma autoantigen 1.
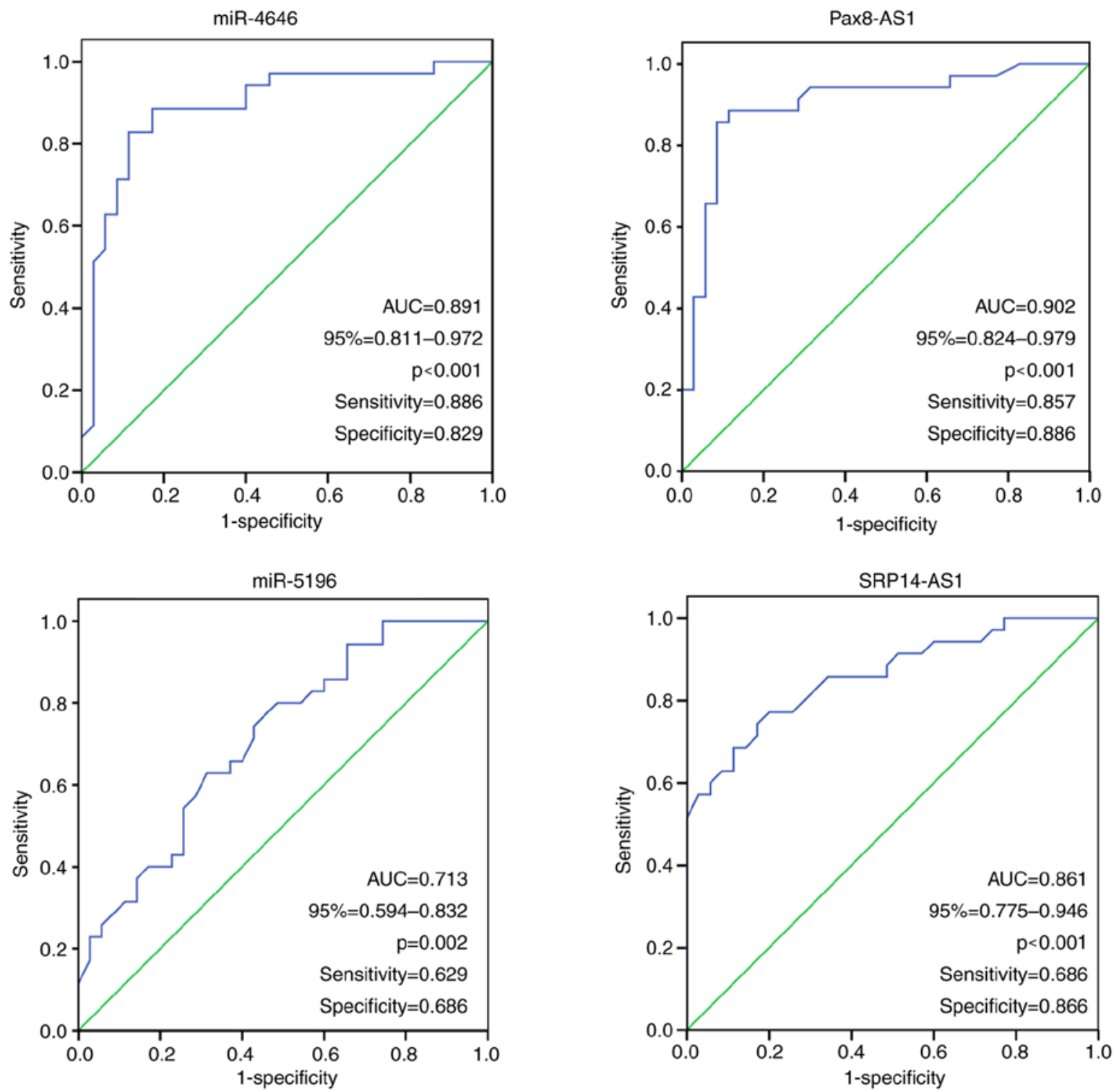

Figure 4. Diagnostic value of non-coding RNAs in the leukocytes of females in the second trimester as markers for gestational diabetes were analysed by receiver operating characteristic curve analysis. miR-4646 cut-off: 1.95; Pax8-AS1 cut-off: 2.165; miR-5196 cut-off: 2.59 and SRP14-AS1 cut-off: 3.97. AUC, area under the curve, provided with 95\% CI; miR, microRNA; Pax8-AS1, paired box 8 antisense 1; IL21R, interleukin 21 receptor; SRP14, signal recognition particle 14; MORC2, MORC family CW-type zinc finger 2; LHX4, LIM homeobox 4; SSSCA1, Sjogren syndrome/scleroderma autoantigen 1. 
with a sensitivity and specificity of 85.7 and $88.6 \%$, respectively. Compared with known biomarkers, such as plasma protein profiling in the second trimester, Pax8-AS1 is more conserved and highly sensitive. The present analysis provided insight for the development of novel biomarkers for GDM.

miRNAs have been indicated to have functional relevance in the development of obesity and different types of diabetes (26-28). Numerous studies have also reported that miRNAs have a key role during GDM progression. For instance, miR-517b, which was associated with GDM among pregnancies with male foetuses only, regulates the expression of TNF superfamily member 15 , a pro-inflammatory, anti-angiogenic cytokine, which may reflect a sex-specific placental response to the maternal immune system (29). Wander et al (30) observed associations between plasma levels of miR-21-3p and GDM in overweight/obese but not in healthy maternal individuals. In the present study, four miRNAs were selected as candidate biomarkers for GDM in a patient cohort. Significantly decreased miR-4646 was detected in the leukocytes of patients with GDM. Further analysis demonstrated that there was a strong correlation between miR-4646 levels and 2-h plasma glucose levels in patients with GDM in the second trimester. To assess the diagnostic value of miR-4646 in GDM, ROC curve analysis was performed. The results suggested that the diagnostic value of miR-4646 for GDM was the highest among all miRNAs tested, with a sensitivity and specificity of 88.6 and $82.9 \%$, respectively. To the best of our knowledge, the present study was the first to report that miR-4646 may function as a potential diagnostic biomarker for GDM.

In conclusion, Pax8-AS1 and miR-4646 were decreased in GDM in the second trimester of pregnancy and are closely associated with glycosylation markers. Although the sample size of the present study was limited, the results enhance the current knowledge on the relationship between GDM and miRNAs/lncRNAs. The present results open up possibilities for the diagnosis of GDM or its treatment by regulating the expression of Pax8-AS1 and miR-4646. In the future, identification of further ncRNAs will undoubtedly enhance the current knowledge of new ncRNA functions, allowing us to better understand the pathogenesis and development of GDM and ultimately facilitate the development of ncRNA-directed diagnostics and therapeutics against this potentially fatal disease.

\section{Acknowledgements}

Not applicable.

\section{Funding}

No funding was received.

\section{Availability of data and materials}

All data generated or analysed during this study are included in this published article.

\section{Authors' contributions}

JH and QC contributed to the design of the study, analysis and interpretation of data and prepared all figures and tables.
HM and LG have substantial contributions to the design of the work. YP, CW, DZ and HD took part in analysing the data. All authors read and approved the final manuscript.

\section{Ethics approval and consent to participate}

The study was approved by the Ethics Committee of Yiwu Maternal and Child Hospital (Jinhua, China) and all subjects provided written informed consent.

\section{Patient consent for publication}

Not applicable.

\section{Competing interests}

The authors declare that they have no competing interests.

\section{References}

1. Binder AM, LaRocca J, Lesseur C, Marsit CJ and Michels KB: Epigenome-wide and transcriptome-wide analyses reveal gestational diabetes is associated with alterations in the human leukocyte antigen complex. Clin Epigenetics 7: 79, 2015.

2. Zhu Y and Zhang C: Prevalence of gestational diabetes and risk of progression to type 2 diabetes: A global perspective. Curr Diab Rep 16: 7, 2016.

3. International Association of Diabetes and Pregnancy Study Groups Consensus Panel, Metzger BE, Gabbe SG, Persson B, Buchanan TA, Catalano PA, Damm P, Dyer AR, Leiva A, Hod M, et al: International association of diabetes and pregnancy study groups recommendations on the diagnosis and classification of hyperglycemia in pregnancy. Diabetes Care 33: 676-682, 2010.

4. Sobngwi E, Boudou P, Mauvais-Jarvis F, Leblanc H, Velho G, Vexiau P, Porcher R, Hadjadj S, Pratley R, Tataranni PA, et al: Effect of a diabetic environment in utero on predisposition to type 2 diabetes. Lancet 361: 1861-1865, 2003.

5. Catalano PM and Hauguel-De Mouzon S: Is it time to revisit the Pedersen hypothesis in the face of the obesity epidemic? Am J Obstet Gynecol 204: 479-487, 2011.

6. El Hajj N, Schneider E, Lehnen H and Haaf T: Epigenetics and life-long consequences of an adverse nutritional and diabetic intrauterine environment. Reproduction 148: R111-R120, 2014.

7. Esteller M: Non-coding RNAs in human disease. Nat Rev Genet 12: 861-874, 2011.

8. Anastasiadou E, Jacob LS and Slack FJ: Non-coding RNA networks in cancer. Nat Rev Cancer 18: 5-18, 2018.

9. Quinn JJ and Chang HY: Unique features of long non-coding RNA biogenesis and function. Nat Rev Genet 17: 47-62, 2016.

10. Yan C, Chen J and Chen N: Long noncoding RNA MALAT1 promotes hepatic steatosis and insulin resistance by increasing nuclear SREBP-1c protein stability. Sci Rep 6: 22640, 2016.

11. Leng L, Zhang C, Ren L and Li Q: Construction of a long non-coding RNA-mediated competitive endogenous RNA network reveals global patterns and regulatory markers in gestational diabetes. Int J Mol Med 43: 927-935, 2019.

12. Tuna M, Machado AS and Calin GA: Genetic and epigenetic alterations of microRNAs and implications for human cancers and other diseases. Genes Chromosomes Cancer 55: 193-214, 2016.

13. Mitchell PS, Parkin RK, Kroh EM, Fritz BR, Wyman SK, Pogosova-Agadjanyan EL, Peterson A, Noteboom J, O'Briant KC, Allen A, et al: Circulating microRNAs as stable blood-based markers for cancer detection. Proc Natl Acad Sci USA 105: 10513-10518, 2008.

14. Esteves JV, Enguita FJ and Machado UF: MicroRNAs-mediated regulation of skeletal muscle GLUT4 expression and translocation in insulin resistance. J Diabetes Res 2017: 7267910, 2017.

15. Pordzik J, Jakubik D, Jarosz-Popek J, Wicik Z, Eyileten C, De Rosa S, Indolfi C, Siller-Matula JM, Czajka P and Postula M: Significance of circulating microRNAs in diabetes mellitus type 2 and platelet reactivity: Bioinformatic analysis and review. Cardiovasc Diabetol 18: 113, 2019. 
16. Stirm L, Huypens P, Sass S, Batra R, Fritsche L, Brucker S, Abele H, Hennige AM, Theis F, Beckers J, et al: Maternal whole blood cell miRNA-340 is elevated in gestational diabetes and inversely regulated by glucose and insulin. Sci Rep 8: 1366, 2018.

17. Livak KJ and Schmittgen TD: Analysis of relative gene expression data using real-time quantitative PCR and the 2(-Delta Delta C(T)) method. Method 25: 402-408, 2001.

18. Iempridee $T$, Wiwithaphon $S$, Piboonprai K, Pratedrat $P$, Khumkhrong P, Japrung D, Temisak S, Laiwejpithaya S, Chaopotong $\mathrm{P}$ and Dharakul T: Identification of reference genes for circulating long noncoding RNA analysis in serum of cervical cancer patients. FEBS Open Bio 8: 1844-1854, 2018.

19. Li C, Chen J and Qin G: Partial Youden index and its inferences. J Biopharm Stat 29: 385-399, 2019.

20. Wapinski $\mathrm{O}$ and Chang HY: Long noncoding RNAs and human disease. Trends Cell Biol 21: 354-361, 2011.

21. $\mathrm{Li} \mathrm{CH}$ and Chen Y: Targeting long non-coding RNAs in cancers: Progress and prospects. Int J Biochem Cell Biol 45: 1895-1910, 2013.

22. Carter G, Miladinovic B, Patel AA, Deland L, Mastorides S and Patel NA: Circulating long noncoding RNA GAS5 levels are correlated to prevalence of type 2 diabetes mellitus. BBA Clin 4: 102-107, 2015

23. Bahari G, Hashemi M, Naderi M, Sadeghi-Bojd S and Taheri M: Long non-coding RNA PAX8-AS1 polymorphisms increase the risk of childhood acute lymphoblastic leukemia. Biomed Rep 8: 184-190, 2018.
24. Han J, Zhou W, Jia M, Wen J, Jiang J, Shi J, Zhang K, Ma H, Liu J, Ren J, et al: Expression quantitative trait loci in long non-coding RNA PAX8-AS1 are associated with decreased risk of cervical cancer. Mol Genet Genomics 291: 1743-1748, 2016.

25. Zhang Y, Li F and Chen J: MYC promotes the development of papillary thyroid carcinoma by inhibiting the expression of lncRNA PAX8-AS1:28. Oncol Rep 41: 2511-2517, 2019.

26. Arner $\mathrm{P}$ and Kulyté A: MicroRNA regulatory networks in human adipose tissue and obesity. Nat Rev Endocrinol 11: 276-288, 2015.

27. Miao C, Chang J, Zhang G and Fang Y: MicroRNAs in type 1 diabetes: New research progress and potential directions. Biochem Cell Biol 96: 498-506, 2018.

28. Vienberg S, Geiger J, Madsen S and Dalgaard LT: MicroRNAs in metabolism. Acta Physiol (Oxf) 219: 346-361, 2017.

29. Anton L, Olarerin-George AO, Hogenesch JB and Elovitz MA: Placental expression of $\mathrm{miR}-517 \mathrm{a} / \mathrm{b}$ and miR-517c contributes to trophoblast dysfunction and preeclampsia. PLoS One 10: e0122707, 2015.

30. Wander PL, Boyko EJ, Hevner K, Parikh VJ, Tadesse MG, Sorensen TK, Williams MA and Enquobahrie DA: Circulating early- and mid-pregnancy microRNAs and risk of gestational diabetes. Diabetes Res Clin Pract 132: 1-9, 2017.

This work is licensed under a Creative Commons Attribution-NonCommercial-NoDerivatives 4.0 International (CC BY-NC-ND 4.0) License. 